\title{
The irregularities of the sunspot cycle and their theoretical modelling
}

\author{
Arnab Rai Choudhuri
}

July 25, 2018

\begin{abstract}
The 11-year sunspot cycle has many irregularities, the most prominent amongst them being the grand minima when sunspots may not be seen for several cycles. After summarizing the relevant observational data about the irregularities, we introduce the flux transport dynamo model, the currently most successful theoretical model for explaining the 11-year sunspot cycle. Then we analyze the respective roles of nonlinearities and random fluctuations in creating the irregularities. We also discuss how it has recently been realized that the fluctuations in meridional circulation also can be a source of irregularities. We end by pointing out that fluctuations in the poloidal field generation and fluctuations in meridional circulation together can explain the occurrences of grand minima.
\end{abstract}

\section{Introduction}

The number of sunspots seen on the solar surface rises and falls with a period of about 11 years. This 11-year cycle of sunspots is one of most intriguing natural cycles which is affecting our lives in many ways as our society becomes more dependent on technology. Violent explosions known as solar flares occur more frequently when there are more sunspots. Apart from producing the beautiful polar aurorae, a large flare can disturb the ionosphere causing disruptions in radio communication, can damage electronics in man-made satellites, can make airlines flights near geomagnetic poles particularly hazardous and can even trip power grids. On 13 March 1989, a large part of eastern Canada had a power blackout caused by a powerful solar flare.

Figure 1 shows the sunspot number as a function of time from 1610. Galileo and some of his contemporaries were the first scientists to study sunspots systematically. The initial entries in Figure 1 are based on their records. Then, for nearly a century, sunspots were rarely seen - a period known as the Maunder minimum. Afterwards the sunspot number has varied periodically with a rough period of about 11 years, although we see a considerable amount of irregularity. Some cycles are stronger than the average and some are weaker. 


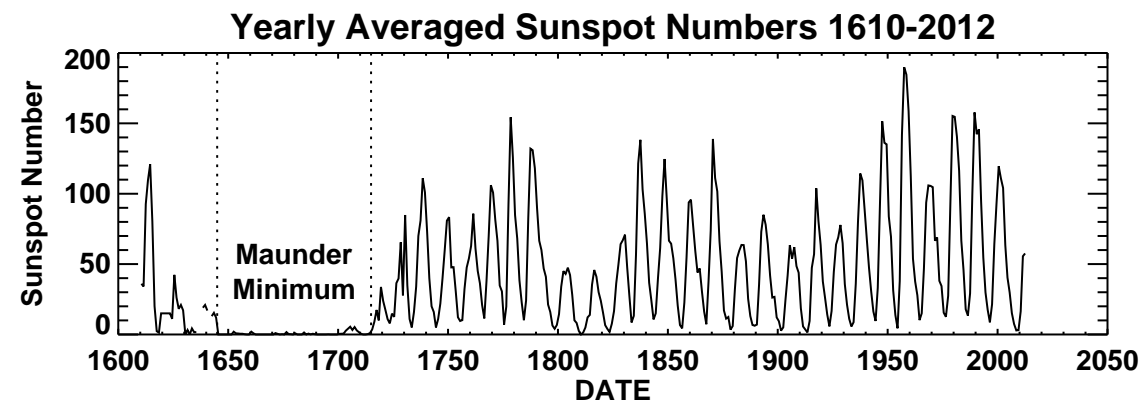

Figure 1: A plot of the yearly averaged sunspot number from 1610 to the present time.

After the discovery of the sunspot cycle by Schwabe in 1843 [1], for a long time there was no theoretical explanation for it. When Hale discovered in 1908 [2] that a sunspot is a site of a concentrated magnetic field (about $0.3 \mathrm{~T}$, only a little bit weaker than the strongest magnetic fields produced in our laboratories by large electromagnets), it became clear that the 11-year sunspot cycle is essentially the magnetic cycle of the Sun. It may be mentioned that Hale's discovery of magnetic fields in sunspots was a truly momentous discovery in the history of physics because this was the first time somebody found a conclusive evidence of large-scale magnetic fields outside the Earth's environment. Now we know that magnetic fields are ubiquitous in the astronomical universe.

It is now generally accepted that a magnetohydrodynamic (MHD) process known as the dynamo process is responsible for generating magnetic fields in astrophysical systems. The foundations of dynamo theory were laid down in a 1955 classic paper by Parker [3], in which he derived the dynamo equation arising out of MHD turbulence subject to rotation. Afterwards, Steenbeck, Krause and Rädler [4] developed the mean field formalism of dynamo theory in a more systematic way.

The particular dynamo process responsible for producing the 11-year sunspot cycle is called the flux transport dynamo process. Invoking some early ideas due to Babcock [5] and Leighton [6], the flux transport dynamo theory was first formulated by Wang, Sheeley and Nash [7], Choudhuri, Schüssler and Dikpati [8] and Durney [9]. This theory has been remarkably successful in providing theoretical explanation of various aspects of the sunspot cycle. At first, efforts were focussed on explaining regular aspects of the sunspot cycle. After the successful modelling of the regular aspects, the thrust of research in the last few years has been to apply the flux transport dynamo model to study the irregularities of the sunspot cycle.

An earlier review by the present author [10] summarized the basic observational data about the sunspot cycle and then discussed how the flux transport dynamo model was developed to explain these observational data. Although we shall briefly summarize the salient features of the flux transport dynamo, we do 
not want to repeat the full discussions of the previous review. So we would urge the readers to read this previous review before reading the present review. The present review can be regarded as a continuation of the previous review. The main aim of the present review will be to discuss how the irregularities of the sunspot cycle are modelled with the flux transport dynamo. Although a little bit of discussion of this subject can be found at the end of the previous review [10], some very important developments took place in this field after that review was written. These very recent developments will be highlighted throughout the present review.

\section{Some aspects of observational data}

The earlier review [10] provided a summary of the regular periodic aspects of the sunspot cycle (Hale's polarity law, butterfly diagram). So we not discuss those topics here. We merely focus our attention on the irregularities of the sunspot cycle. If all the irregularities were really 'irregular' in the true sense, then it would have been very difficult to develop any theoretical understanding about them. However, one can discern certain patterns within the irregularities which give us valuable clues how these irregularities may arise and how they can be modelled theoretically.

To discover patterns within the irregularities of the sunspot cycle, one would like to have as much data about the irregularities as possible, so that statistical inferences become meaningful. We have actual sunspot records for about four centuries, although the records become less reliable as we go earlier than the nineteenth century. One important question is whether we have other proxies of sunspot activity through which we can infer about sunspot cycles in the past even without actual sunspot records. When the sunspot activity is low, the magnetic field in the solar wind becomes weaker, allowing more cosmic ray particles to reach the Earth and to produce more of the radioactive nuclei ${ }^{10} \mathrm{Be}$ and ${ }^{14} \mathrm{C}$ by interacting with air molecules. If we can infer what the concentrations of ${ }^{10} \mathrm{Be}$ and ${ }^{14} \mathrm{C}$ in the atmosphere were at earlier times, then from that we can reconstruct a history of sunspot cycles in the past. The atmospheric concentration of ${ }^{14} \mathrm{C}$ in the past can be inferred by analyzing old tree rings, whereas the atmospheric concentration of ${ }^{10} \mathrm{Be}$ in the past can be inferred from the polar ice cores which have formed over many years. It has now been possible to reconstruct the history of sunspot activity for the past 11,000 years.

At the first sight, the strengths of different sunspot cycles as seen in Figure 1 may appear to vary randomly. Let us first discuss if there are any long-term patterns. Sunspot cycles have been numbered from the middle of the eighteenth century, the present cycle being cycle 24 . For several cycles from cycle 10, the odd cycle has been stronger than the previous even cycle, a pattern at last broken by cycle 23 which turned out to be weaker than cycle 22. This is called the Gnevyshev-Ohl rule [11], though departures from this rule are known. Apart from this two-cycle pattern, it is often claimed that there a modulation of cycle amplitudes involving eight cycles, often called the Gleissberg cycle. From the 
limited data we have, it is very difficult to either prove or disprove the existence of the Gleissberg cycle. What is clear, however, is that sometimes the sunspot activity may almost disappear for many years and several cycles may go missing, like what happened during the Maunder minimum. Such events are called grand minima. On reconstructing the sunspot activity for several millenia, it is now clear that the Maunder minimum was not unique. It is estimated that there have been 27 such grand minima during the last 11,000 years [12].

Apart from these patterns involving the amplitudes of different cycles, there are other patterns within the irregularities of sunspot cycles. The earlier review [10] discussed in detail the possible correlation between the polar fields during the sunspot minima and the strengths of next cycles. If such a correlation does exist (which seems to be the case from the limited data we have), then that gives a powerful tool for predicting the strength of a sunspot cycle before its beginning, once we know the strength of the polar field during the previous sunspot minimum. The last interesting pattern to which we wish to draw the readers' attention is what is called the Waldmeier effect [13]. It appears that strong cycles rise fast, whereas weak cycles rise more slowly. In other words, there is an anti-correlation between the rise times of the cycles and their strengths.

Within the last few years, attempts are being made to explain these patterns of irregularities with the flux transport dynamo model. After discussing the

basic model in the next section, we shall come to the theoretical modelling of irregularities from $\S 3$.

\section{$3 \quad$ Flux transport solar dynamo}

We now give a very brief summary of the flux transport dynamo model of the sunspot cycle. We emphasize again that this discussion is not meant to be selfexplanatory. It is not meant to be accessible to readers without any previous knowledge of the subject. Readers without any previous knowledge are urged to read the previous review [10] before proceeding further.

The toroidal and the poloidal components of the Sun's magnetic field are supposed to sustain each other through a feedback loop. The differential rotation of the Sun (which is now fully mapped by helioseismology) stretches out the poloidal field to produce the toroidal field. This primarily takes place at the bottom of the solar convection zone (at $r=0.7 R_{\odot}$ ) where the differential rotation is concentrated. To complete the dynamo loop, the poloidal field has to be generated back from this toroidal field. How this happens is more subtle. The original idea of Parker [3] and Steenbeck, Krause and Rädler [4] —often called the $\alpha$-effect - was that the toroidal field is twisted by the helical turbulence of the convection zone to produce the poloidal field. This is possible only if the toroidal field does not have energy density more than the energy density of turbulence. The condition for this is that the toroidal field should not be stronger than $10^{4} \mathrm{G}$. The idea of the toroidal field being twisted by helical turbulence had to be questioned when detailed calculations of the rise of the toroidal field by magnetic buoyancy to form sunspots were out on the basis of 


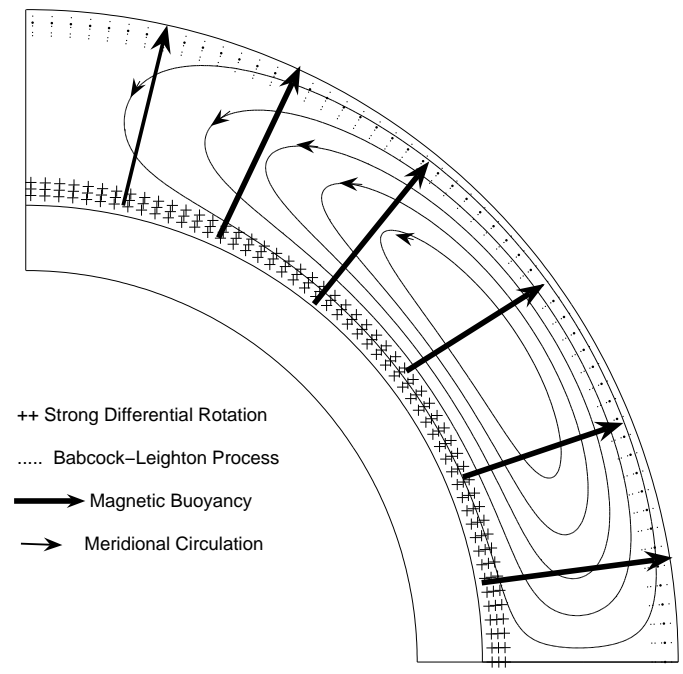

Figure 2: A cartoon explaining how the flux transport dynamo works.

the thin flux tube equation [14-15]. The simulations of Choudhuri and Gilman [16], Choudhuri [17], D'Silva and Choudhuri [18] and Fan, Fisher and DeLuca [19] suggested that the toroidal field at the bottom of the convection zone has to be as strong as $10^{5} \mathrm{G}$ in order to match different aspects of observations. The $\alpha$-effect cannot operate on such a strong field.

An alternative idea of the poloidal field generation goes back to Babcock [5] and Leighton [6]. The toroidal field rising due to magnetic buoyancy produces bipolar sunspots on the solar surface with tilts caused by the Coriolis forcean effect known as Joy's law. When a tilted bipolar sunspot decays, the two opposite magnetic polarities spread preferentially in slightly different latitudes. Many of us now believe that the poloidal field generation in the solar dynamo takes place due to this Babcock-Leighton mechanism. The Sun has a meridional circulation which is poleward near the surface and advects this poloidal field poleward [20-23]. This meridional circulation also plays a crucial role in the solar dynamo. The kind of dynamo in which the poloidal field is generated by the Babcock-Leighton mechanism and the meridional circulation plays a critical role is called the flux transport dynamo.

Figure 2 is a cartoon explaining how the flux transport dynamo operates within the solar convection zone. The toroidal field is generated at the bottom of the convection zone where the strong differential rotation discovered by helioseismology stretches out the poloidal field to generate the toroidal field. Then this toroidal field rises to the solar surface due to magnetic buoyancy to produce the tilted bipolar sunspots. The decay of these tilted bipolar sunspots then gives rise to the poloidal field near the surface by the Babcock-Leighton mechanism. The meridional circulation is also indicated in Figure 2. We observe 
the meridional circulation to be poleward in the top layers of the convection zone. In order to conserve mass, the meridional circulation has to be equatorward deeper down. It is generally assumed in flux transport dynamo models that the equatorward flow is at the bottom of the convection zone, although this is not yet confirmed from observations. The poloidal field produced near the surface is advected poleward by the poleward meridional circulation there, whereas the toroidal field produced at the bottom of the convection zone is advected equatorward by the equatorward meridional circulation there. This provides the theoretical explanation of both the observed poleward drift of the surface magnetic field (outside active regions) and the equatorward migration of the sunspots which form from the toroidal field. While the basic idea of the flux transport dynamo was given in an early paper by Wang, Sheeley and Nash [7], the first two-dimensional models were constructed by Choudhuri, Schüssler and Dikpati [8] and Durney [9].

A numerical code SURYA was developed in our group in Indian Institute of Science to solve the basic equations of the flux transport dynamo [24-25] and was made public from 2005. A comparison of the observational data shown in Figure 2 [10] with theoretical results of [25] shown in Figure 10 makes it clear that the flux transport dynamo is reasonably successful in reproducing various aspects of the periodic behaviour of the sunspot cycle. Apart from solving the solar dynamo problem, the code SURYA has also been modified to study the accretion of matter on magnetized neutron stars [26-27]. It may be noted that a flux tube approach has to be combined with the mean field dynamo equation to have a more complete understanding of the magnetic field dynamics within the solar convection zone [28]. For example, we have to consider the wrapping of poloidal field lines around rising flux tubes to explain how the observed current helicity of sunspots arise [29-30]. The flux transport dynamo model has also been applied to model the back-reactions of the dynamo-generated magnetic field such as torsional oscillations [31].

There have been some recent claims that the equatorward reverse flow of the meridional circulation occurs at a shallow depth and not at the bottom of the convection zone as usually assumed in the flux transport dynamo model [32-33]. If these claims are corroborated by other independent studies and turn out to be true, then we have to address the question whether the flux transport dynamo can work with a shallow meridional circulation. Guerrero and de Gouveia Dal Pino [34] considered a shallow cell of meridional circulation with equatorward turbulent pumping in the region below it and succeeded in getting realistic butterfly diagrams. Whether such latitudinal pumping exists is questionable. If there is just a shallow cell of meridional circulation and nothing below it, then the flux transport dynamo cannot work. However, recently Hazra, Karak and Choudhuri [35] showed that many of the attractive features of the flux transport dynamo are retained if, below the shallow cell of meridional circulation at the top of the convection zone, there are additional cells such that there is an equatorward meridional circulation at the bottom of the convection zone. Thus, even if the meridional circulation has a return flow at a shallow depth, the flux transport dynamo can presumably still work as long as there is an appropriate 
equatorward flow at the bottom of the convection zone.

The original flux transport dynamo model of Choudhuri, Schüssler and Dikpati [8] led to two offsprings: a high diffusivity model and a low diffusivity model. The diffusion times in these two models are of the order of 5 years and 200 years respectively. The high diffusivity model has been developed by a group working in IISc Bangalore (Choudhuri, Nandy, Chatterjee, Jiang, Karak), whereas the low diffusivity model has been developed by a group working in HAO Boulder (Dikpati, Charbonneau, Gilman, de Toma). The differences between these models have been systematically studied by Jiang, Chatterjee and Choudhuri [36] and Yeates, Nandy and Mckay [37]. Both these models are capable of giving rise to oscillatory solutions resembling solar cycles. However, when we try to study the irregularities of the cycles, the two models give completely different results. We need to introduce fluctuations to cause irregularities in the cycles. In the high diffusivity model, fluctuations spread all over the convection zone in about 5 years. On the other hand, in the low diffusivity model, fluctuations essentially remain frozen during the cycle period. Thus the behaviours of the two models are totally different on introducing fluctuations. As we shall see in the next three Sections, only the high diffusivity model can provide explanations for certain aspects of sunspot cycle irregularities. The high diffusivity also helps in establishing the dipolar parity of the solar magnetic field $[25,38]$ and can explain the lack of significant hemispheric asymmetry [39-40].

\section{Nonlinearities versus random fluctuations}

The magnetic fields produced by the dynamo can react back on the velocity fields driving the dynamo action. This leads to nonlinearities in the mathematical theory. It is well known that nonlinear dynamical systems can show complicated chaotic behaviours and one possibility is that irregularities of the sunspot cycle are just a manifestation of such chaotic behaviour. However, the mean field theory of the dynamo involves averaging over turbulence and we always have fluctuations around the mean. These random fluctuations also may be the source of irregularities. For some time, there has been a debate in this field whether the irregularities of the sunspot cycle are primarily due to nonlinear chaos or due to random fluctuations. While we now think that there are signatures of both the effects, it seems that the really large irregularities like the grand minima are caused by random fluctuations.

Let us point out why many of us think that the largest irregularities of the sunspot cycle are not due to nonlinear chaos. The simplest way of capturing the effect of the nonlinear feedback in a kinematic dynamo model (in which the fluid equations are not solved) is to consider a quenching of the $\alpha$ parameter (the crucial parameter in the dynamo generation of magnetic fields) as follows:

$$
\alpha=\frac{\alpha_{0}}{1+\left|\bar{B} / B_{0}\right|^{2}},
$$

where $\bar{B}$ is the mean magnetic field produced by the dynamo and $B_{0}$ is the value 
of magnetic field beyond which nonlinear effects become important. There is a long history of dynamo models studied with such quenching [41-43]. In most of the nonlinear calculations, however, the dynamo eventually settles to a periodic mode with a given amplitude rather than showing sustained irregular behaviour. The reason for this is intuitively obvious. Since a sudden increase in the amplitude of the magnetic field would diminish the dynamo activity by reducing $\alpha$ given by (2) and thereby pull down the amplitude again (a decrease in the amplitude would do the opposite), the $\alpha$-quenching mechanism tends to lock the system to a stable mode once the system relaxes to it. Only by using somewhat unusual kinds of nonlinearities, usually with large time delays, it is sometimes possible to get chaotic behaviour in the system. Although nonlinearities may not produce sustained chaotic behaviour, It has been suggested that the Gnevyshev-Ohl rule is caused by a period doubling due to nonlinearities [44-45] and there is no other good theoretical explanation for it. Presumably the nonlinearities play some role in producing such effects as the GnevyshevOhl rule, but we believe that they are not the main cause behind the large irregularities of the sunspot cycle.

Now let us come to the possibility that the irregularities of the sunspot cycle are primarily caused by random fluctuations, as suggested first by Choudhuri [46] and Hoyng [47]. The crucial issue is to figure out the nature of random fluctuations in the flux transport dynamo. Choudhuri, Chatterjee and Jiang [48] identified the Babcock-Leighton mechanism of poloidal field generation as the main source of random fluctuations. This mechanism depends on the tilts of bipolar sunspot pairs. While the average tilts are given by Joy's law, one finds a large scatter around this average, presumably produced by the fact that the rising flux tubes are buffeted by turbulence in the convection zone[49]. This scatter around Joy's law produces fluctuations in the poloidal field generation process, ultimately giving rise to irregularities in the dynamo mechanism. In the high diffusivity flux transport dynamo model, we can theoretically explain the observed correlation between the polar field during the sunspot minimum and the strength of the next cycle if the irregularities of cycles primarily arise due to fluctuations in the Babcock-Leighton mechanism, but we do not get this correlation in the low diffusivity model [36]. Since the origin of this correlation in high diffusivity model has been discussed in detail in the earlier review [10], we shall not get into a detailed discussion of this subject here, except to mention that the theoretical explanation of this correlations lends support simultaneously to the high diffusivity dynamo model and to the idea that the fluctuations in the Babcock-Leighton mechanism is the major cause of irregularities in the sunspot cycle. Recent analyses of the sunspot tilt data by different groups also provide strong support to the scenario outlined above [50-51].

We have already mentioned that the correlation between the polar field during a sunspot minimum and the strength of the next cycle provides a mechanism for predicting future cycles. We shall only make some comments on this. On the basis of the observation that the polar field was rather weak during the last sunspot minimum, several groups predicted a few years ago that the present cycle 24 would be rather weak [52-53]. One crucial question at that time was 


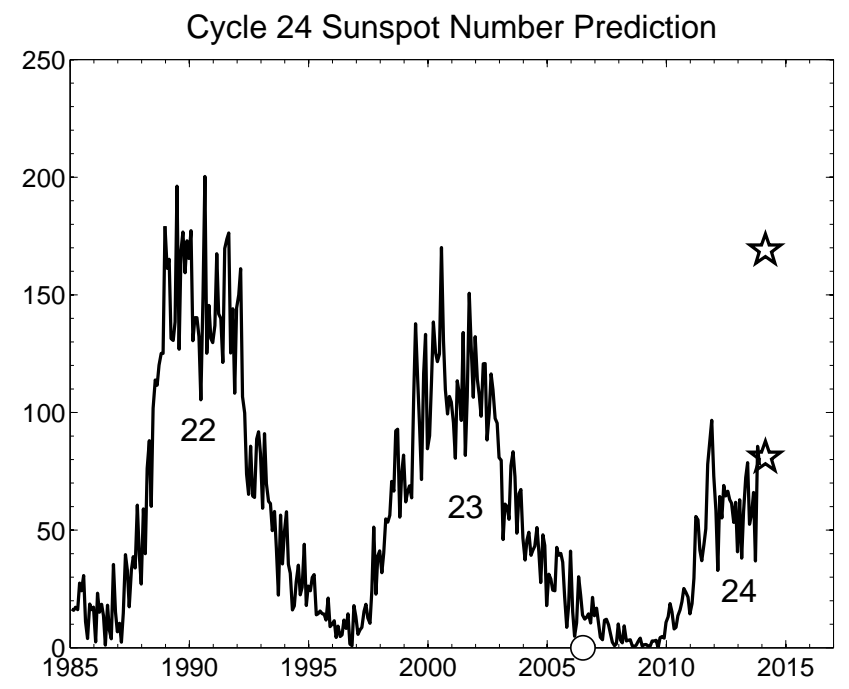

Figure 3: The monthly sunspot number plot for the last few years, indicating the theoretical predictions. The upper star is the peak of cycle 24 predicted by Dikpati and Gilman [54], whereas the lower star is what was predicted by Choudhuri, Chatterjee and Jiang [48]. The circle on the horizontal axis indicates the time when these predictions were made (in 2006).

whether theoretical solar dynamo models could be used to make a prediction. During the sunspot minimum before the previous cycle 23 (in the mid-1990s), solar dynamo models were still too primitive for this purpose. The sunspot minimum before the present cycle 24 was the first sunspot minimum during which the solar dynamo models has reached a certain level of sophistication to make such predictions. Dikpati and Gilman [54] used their low diffusivity model to predict that the cycle 24 would be the strongest cycle in the last half century. On the other hand, Choudhuri, Chatterjee and Jiang [48] used their high diffusivity model to predict that the cycle 24 will be the weakest cycle in nearly a century. This is a rather robust prediction of this high diffusivity model, because this model produces a strong correlation between the polar field during the sunspot minimum and the next cycle, and the fact that the polar field was very weak during the last sunspot minimum was incorporated in the theoretical model for this prediction work. Figure 3 shows the present status of the sunspot number data with the two theoretical predictions indicated. It is clear that the observational data is consistent with the prediction of Choudhuri, Chatterjee and Jiang [48], making this the first successful prediction of a cycle from a theoretical dynamo model in the history of this subject.

Lastly, we come to the question whether fluctuations in the poloidal field generation can produce grand minima. When the poloidal field at the end of a 
cycle falls to a very low value due to these fluctuations, Choudhuri and Karak [55] found that the dynamo can be pushed into a grand minimum. In fact, they were able to construct an example of a grand minimum having the broad features of the Maunder minimum. We thus conclude that the fluctuations in the Babcock-Leighton mechanism for generating the poloidal field is a possible mechanism for producing grand minima - especially if the dynamo is not too supercritical [56].

\section{$5 \quad$ Fluctuations in meridional circulation}

Until about 5-6 years ago, it was not generally recognized that there is another important source of sunspot cycle irregularities: fluctuations in the meridional circulation. It is well known that the period of the flux transport dynamo varies roughly as the inverse of the meridional circulation speed. The period of the dynamo is approximately given by the time taken by meridional circulation at the bottom of the convection zone to move from higher latitudes to lower latitudes. Since the meridional circulation determines the period of the flux transport dynamo, it is not surprising that any fluctuations in meridional circulation would have an effect on the flux transport dynamo. It has been found recently that the meridional circulation has a periodic variation with the solar cycle, becoming weaker at the time of sunspot maximum [57-59]. Presumably the Lorentz force of the dynamo-generated magnetic field slows down the meridional circulation at the time of the sunspot maximum. Karak and Choudhuri [60] found that this quenching of meridional circulation by the Lorentz force does not produce irregularities in the cycle, provided the diffusivity is high as we believe. Then the question arises whether there are other kinds of fluctuations in the meridional circulation apart from these cyclic modulations.

We have reliable observational data on the variation of meridional circulation only for a little more than a decade. To draw any conclusions about the variation of meridional circulation at earlier times, we have to rely on indirect arguments. If we assume the cycle period to go inversely as meridional circulation, then we can use periods of different past solar cycles to infer how meridional circulation has varied with time in the last few centuries. On the basis of such considerations, it appears that the meridional circulation had random fluctuations in the last few centuries with correlation time of the order of 30-40 years [61]. We now come to question what effect these random fluctuations of meridional circulation may have on the dynamo. Based on the analysis of Yeates, Nandy and Mckay [37], we can easily see that dynamos with high and low diffusivity will be affected very differently. Suppose the meridional circulation has suddenly fallen to a low value. This will increase the period of the dynamo and lead to two opposing effects. On the one hand, the differential rotation will have more time to generate the toroidal field and will try to make the cycles stronger. On the other hand, diffusion will also have more time to act on the magnetic fields and will try to make the cycles weaker. Which of these two competing effects wins over will depend on the value of diffusivity. If the diffusivity is high, then the 
action of diffusivity is more important and the cycles become weaker when the meridional circulation is slower. The opposite happens if the diffusivity is low.

The important question now is if there is any kind of observational data to indicate whether the cycles become weaker (which will happen for high diffusivity) or stronger (which will happen for low diffusivity) when the meridional circulation is slower. The Waldmeier effect discussed in $\S 2$ provides precisely this kind of observational data. The rise time of the sunspot cycle roughly goes as the duration of the cycle. If the meridional circulation is slower, then the cycle is longer and the rise time is also longer. According to the Waldmeier effect, the longer cycle tends to be weaker in strength. This happens only if the turbulent diffusivity is high. Karak and Choudhuri [61] were able to explain the Waldmeier effect on the basis of the high diffusivity model, whereas the low diffusivity would give the opposite of the Waldmeier effect. The success in explaining the Waldmeier effect is another feather in the cap of the high diffusivity model.

Since a slowing of the meridional circulation would make the cycles weaker, a question that comes before us is whether a sufficient slowing of the meridional circulation can cause a grand minimum. Karak [62] indeed found that the flux transport dynamo can be pushed into a grand minimum if the meridional circulation drops to 0.4 of its normal value. This is clearly another possible mechanism for producing a grand minimum.

\section{A theoretical model of grand minima}

From the discussions in the previous two sections, it should be clear that a grand minimum can be caused by two means: if the poloidal field produced at the end of cycle is very weak as a result of fluctuations in the Babcock-Leighton mechanism and if the meridional circulation falls to a very low value due to its

fluctuations. Presumably the grand minima arise due to the combined effect of both these kinds of fluctuations, as shown by Choudhuri and Karak [63]. Let $\gamma$ be the normalized strength of the polar field (i.e. the strength of the polar field divided by its average value over many cycles) at the end of a cycle and let $v_{0}$ be the amplitude of the meridional circulation. Figure 4 shows the twodimensional parameter space of $\gamma$ versus $v_{0}$. The condition at the beginning of a sunspot cycle is clearly represented by a point in this two-dimensional parameter space. Choudhuri and Karak [63] found that the dynamo is pushed into a grand minimum if the condition at the beginning of the cycle corresponds to the shaded region of the parameter space. What is the probability of this happening?

Presumably both the fluctuations we are considering would be of Gaussian nature. Then the joint probability that the polar field strength at the end of a cycle lies in the range $\gamma, \gamma+d \gamma$ and the amplitude of the meridional circulation 


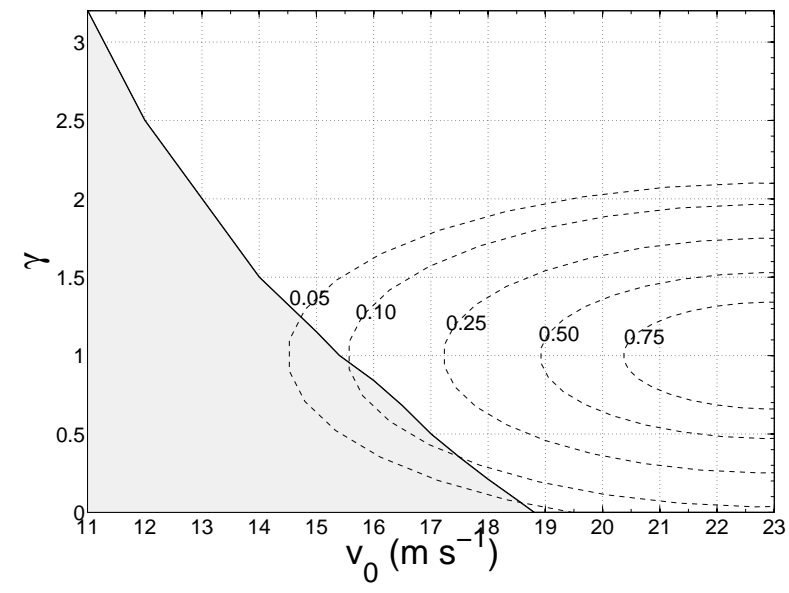

Figure 4: The parameter space indicating the normalized strength $\gamma$ of the poloidal field and the amplitude of the meridional circulation, the shaded region being the part to the parameter space giving rise to grand minima.

at the same time lies in the range $v_{0}, v_{0}+d v_{0}$ is given by

$$
P\left(\gamma, v_{0}\right) d \gamma d v_{0}=\frac{1}{\sigma_{v} \sqrt{2 \pi}} \exp \left[-\frac{\left(v_{0}-\overline{v_{0}}\right)^{2}}{2 \sigma_{v}^{2}}\right] \frac{1}{\sigma_{\gamma} \sqrt{2 \pi}} \exp \left[-\frac{(\gamma-1)^{2}}{2 \sigma_{\gamma}^{2}}\right] d \gamma d v_{0} .
$$

The probability that the condition at the beginning of a cycle lies in the shaded region of Figure 4 is obtained by integrating the double Gaussian given by (2) over this region. To carry on this integration, we need values of $\sigma_{v}$ and $\sigma_{\gamma}$, which are the widths of these Gaussians. Choudhuri and Karak [63] realized that these can be obtained from the observational data of the last 28 cycles. The periods of these cycles give the values of the meridional circulation during these cycle, from which the probability distribution function of the meridional circulation can be constructed. Since strengths of the cycles are correlated with the polar field strength $\gamma$ at the beginning of the cycle, the strengths of the last 28 cycles can be used to construct the probability distribution function of $\gamma$. Although we would not expect a very good Gaussian fit from a set of 28 data points, Choudhuri and Karak [63] found that the fits were not too bad and could estimate the values of $\sigma_{v}, \sigma_{\gamma}$. On carrying out the integration of the double Gaussian over the shaded region in Figure 4, Choudhuri and Karak [63] found the value to be $1.7 \%$. This means that 17 cycles out of 1000 cycles (in 11,000 years) would have conditions appropriate for grand minima at their beginnings. This is remarkably close to the observational data that there had been 27 grand minima in the last 11,000 years. In fact, in actual runs of the dynamo code with fluctuations given by the double Gaussian (2), Choudhuri and Karak [63] typically found about 24-30 grand minima in a run spanning 11,000 years. 
While this may seem like a very encouraging result, one aspect of grand minima still remains completely shrouded in mystery. If there are no sunspots at all during a grand minimum, one important question is whether the BabcockLeighton mechanism which depends on the existence of tilted bipolar sunspots can operate at all. If the Babcock-Leighton mechanism is not operative, then some mechanism has to build up the poloidal field so that the Sun can eventually come out of the grand minimum. If the magnetic field during the grand minimum becomes sufficiently weak, then one possibility is that the $\alpha$-effect originally envisaged by Parker [3] and Steenbeck, Krause and Rädler [4] becomes operative. Karak and Choudhuri [64] have done some explorations of this. The results are inconclusive. While we now have some idea how the Sun gets pushed into grand minima, we have very little understanding how the Sun gets out of a grand minimum after falling into one.

\section{Acknowledgement}

I thank DST for partial support through a J C Bose Fellowship.

\section{References}

[1] S H Schwabe, Astron. Nachr. 21, 233 (1844)

[2] G E Hale, Astrophys. J. 28, 315 (1908)

[3] E N Parker, Astrophys. J. 122, 293 (1955)

[4] M Steenbeck, F Krause and K-H Rädler, Z. Naturforsch. 21a, 1285 (1966)

[5] H W Babcock, Astrophys. J. 133, 572 (1961)

[6] R B Leighton, Astrophys. J. 156, 1 (1969)

[7] Y-M Wang, N R Sheeley and A G Nash, Astrophys. J. 383, 431 (1991)

[8] A R Choudhuri, M Schüssler and M Dikpati, Astron. Astrophys. 303, L29 (1995)

[9] B R Durney, Solar Phys. 160, 213 (1995)

[10] A R Choudhuri, Pramana 77, 77 (2011)

[11] M N Gnevyshev and A I Ohl, Astron. Zh. 25, 18 (1948)

[12] I G Usoskin, S K Solanki and G A Kovaltsov, Astron. Astrophys. 471, 301 (2007)

[13] M Waldmeier, Mitt. Eidgen. Sternw. Zurich 14, 105 (1935)

[14] H C Spruit, Astron. Astrophys. 98, 155 (1981) 
[15] A R Choudhuri, Astron. Astrophys. 239, 335 (1990)

[16] A R Choudhuri and P A Gilman, Astrophys. J. 316, 788 (1987)

[17] A R Choudhuri, Solar Phys. 123, 217 (1989)

[18] S D'Silva and A R Choudhuri, Astron. Astrophys. 272, 621 (1993)

[19] Y Fan, G H Fisher and E E DeLuca, Astrophys. J. 405, 390 (1993)

[20] Y-M Wang, A G Nash and N R Sheeley, Astrophys. J. 347, 529 (1989)

[21] M Dikpati and A R Choudhuri, Astron. Astrophys. 291, 975 (1994)

[22] M Dikpati and A R Choudhuri, Solar Phys. 161, 9 (1995)

[23] A R Choudhuri and M Dikpati, Solar Phys. 184, 61 (1999)

[24] D Nandy and A R Choudhuri, Science 296, 1671 (2002)

[25] P Chatterjee, D Nandy and A R Choudhuri, Astron. Astrophys. 427, 1019 (2004)

[26] A R Choudhuri and S Konar, Mon. Notic. Roy. Astron. Soc. 332, 933 (2002)

[27] S Konar and A R Choudhuri, Mon. Notic. Roy. Astron. Soc. 348, 661 (2004)

[28] A R Choudhuri, Solar Phys. 215, 31 (2003)

[29] A R Choudhuri, P Chatterjee and D Nandy, Astrophys. J. 615, L57 (2004)

[30] P Chatterjee, A R Choudhuri and K Petrovay, Astron. Astrophys. 449, 781 (2006)

[31] S Chakraborty, A R Choudhuri and P Chatterjee, Phys. Rev. Lett. 102, $041102(2007)$

[32] D H Hathaway, Astrophys. J. 760, 84 (2012)

[33] J Zhao, R S Bogart, A G Kosovichev, T L Duvall and T Hartlep, Astrophys. J. 774, L29 (2013)

[34] G Guerrero and E M de Gouveia Dal Pino, Astron. Astrophys. 485, 267 (2008)

[35] G Hazra, B B Karak and A R Choudhuri, Astrophys. J. submitted, arXiv:1309.2838 (2014)

[36] J Jiang, P Chatterjee and A R Choudhuri, Mon. Notic. Roy. Astron. Soc. 381, 1527 (2007) 
[37] A R Yeates, D Nandy and D H Mackay, Astrophys. J. 673, 544 (2008)

[38] H Hotta and T Yokoyama, Astrophys. J. 714, L308 (2010)

[39] P Chatterjee and A R Choudhuri, Solar Phys. 239, 29 (2006)

[40] A Goel and A R Choudhuri, Res. Asron. Astrophys. 9, 115 (2009)

[41] M Stix, Astron. Astrophys. 20, 9 (1972)

[42] T S Ivanova and A A Ruzmaikin, Soviet Astron. 21, 479 (1977)

[43] H Yoshimura, Astrophys. J. 226, 706 (1978)

[44] P Charbonneau, C St-Jean and P Zacharias, Astrophys. J. 619, 613 (2005)

[45] P Charbonneau, G Beaubien and C St-Jean, Astrophys. J. 658, 657 (2007)

[46] A R Choudhuri, Astron. Astrophys. 253, 277 (1992)

[47] P Hoyng, Astron. Astrophys. 272, 321 (1993)

[48] A R Choudhuri, P Chatterjee and J Jiang, Phys. Rev. Lett. 98, 131103 (2007)

[49] D Longcope and A R Choudhuri, Solar Phys. 205, 63 (2002)

[50] M Dasi-Espuig, S K Solanki, N A Krivova, R Cameron and T Peñuela, Astron. Astrophys. 518, 7 (2010)

[51] L L Kitchatinov and S V Olemskoy, Astronomy Letters 37, 656 (2011)

[52] L Svalgaard, E W Cliver and Y Kamide, Geo. Res. Lett. 32, L01104 (2005)

[53] K Schatten, Geo. Res. Lett. 32, L21106 (2005)

[54] M Dikpati and P A Gilman, Astrophys. J. 649, 498 (2006)

[55] A R Choudhuri and B B Karak, Res. Asron. Astrophys. 9, 953 (2009)

[56] S V Olemskoy, A R Choudhuri and L L Kitchatinov, Astronomy Reports $\mathbf{5 7}, 458(2013)$

[57] D-Y Chou and D-C Dai, Astrophys. J. 559, L175 (2001)

[58] D H Hathaway and L Rightmire, 2010, Science 327, 1350 (2010)

[59] S Basu and H M Antia, 2010, Astrophys. J. 717, 488 (2010)

[60] B B Karak and A R Choudhuri, Solar Phys. 278, 137 (2012)

[61] B B Karak and A R Choudhuri, Mon. Notic. Roy. Astron. Soc. 410, 1503 (2011)

[62] B B Karak, Astrophys. J. 724, 1021 (2010) 
[63] A R Choudhuri and B B Karak, Phys. Rev. Lett. 109, 171103 (2012)

[64] B B Karak and A R Choudhuri, Res. Asron. Astrophys. 13, 1339 (2013) 